\title{
An Empirical Analysis Over the Four Different Feature-Based Face and Iris Biometric Recognition Techniques
}

\author{
Deepak Sharma \\ Department of CSE \& IT \\ Kurukshetra Institute of Technology \& Management \\ Kurukshetra, Haryana, India
}

\author{
Dr. Ashok Kumar \\ Department of Computer Science Applications \\ Maharishi Markandeshwar University (MMU) \\ Mullana, Ambala, Haryana, India
}

\begin{abstract}
Recently, multimodal biometric systems have been widely accepted, which has shown increased accuracy and population coverage, while reducing vulnerability to spoofing. The main feature to multimodal biometrics is the amalgamation of different biometric modality data at the feature extraction, matching score, or decision levels. Recently, a lot of works are presented in the literature for multi-modal biometric recognition. In this paper, we have presented comparative analysis of four different feature extraction approaches, such as LBP, LGXP, EMD and PCA. The main steps involved in such four approaches are: 1) Feature extraction from face image, 2) Feature extraction from iris image and 3) Fusion of face and iris features. The performance of the feature extraction methods in multi-modal recognition is analyzed using FMR and FNMR to study the recognition behavior of these approaches. Then, an extensive analysis is carried out to find the effectiveness of different approaches using two different databases. The experimental results show the equal error rate of different feature extraction approaches in multi-modal biometric recognition. From the ROC curve plotted, the performance of the LBP and LGXP method is better compared to PCA-based technique.
\end{abstract}

Keywords- Multi-modal biometrics; Face Recognition; iris recognition; LBP operator (Local Binary Pattern); Local Gabor XOR Patterns; PCA and EMD.

\section{INTRODUCTION}

Over the past decade, biometric authentication has drawn substantial attention with growing demands in automated personal identification [4]. This is due to the reason that traditional automatic personal identification systems use tools such as Personal Identification Number (PIN), ID card, key, etc., to verify the identity of a person. But in modern world, such tools are not reliable enough to fulfill the security necessity of person authentication system [5]. A biometric system provides automatic identification of a person by considering some unique features or traits obtained by the person [6]. Some common biometric features are fingerprints, hand-geometry, face, voice, iris, retina, gait, signature, palmprint, ear [5]. An excellent biometric can be identified by use of a feature i.e., the feature should be highly unique - so that the chance of any two person containing the same feature will be negligible, stable - so that the trait does not differ over time, and be easily captured - in order to provide expediency to the user, and prevent falsification of the feature [6].
Multimodal biometric systems are capable of defeating some of the restrictions of unimodal biometric systems by adding multiple sources of information for the process of personal recognition [7]. Such systems are expected to be more dependable because of the existence of several, autonomous fragments of evidence [8,9].

Face recognition is one of the most commonly used biometric features [11]. The intent of face recognition is to identify the persons in images or videos from their facial expression. When a comparison is done with other biometrics, it is found that the face recognition is passive and does not necessitate cooperative persons who are close to or in contact with a sensor [10]. The face is an easily collectible, universal and non-intrusive biometric [12], which makes it perfect for applications while other biometrics such as fingerprints or iris scanning are not possible.

Among the present biometric features, iris is one of the most reliable and precise biometric trait. Iris recognition is widely recognized as one of the preeminent biometrics recognition techniques in the world due to its constancy, distinctiveness, and non-invasiveness, which also has the potential of applications in wide areas [13] [14]. Iris is an externally visible, yet protected organ whose unique epigenetic pattern stays constant throughout the adult life [15]. These features make it suitable for use as a biometric for identifying the persons. Image processing methods are utilized to get the unique iris pattern from a digitized image of the eye, and encrypt it into a biometric template, which can be stored in a database [16]. This biometric template has an objective mathematical representation of the unique data stored in the iris, and permits comparisons to be done among the templates [15]. In iris recognition system, for identifying a person, initially his/her eye is photographed, and then a template is created for their iris region [16]. This template is then compared with the other templates stored in a database. If the matching template is found, the subject is identified or else, the subject remains unidentified [17].

In unimodal biometric systems, only a single feature is employed for validating the identity of a person and such systems are mostly affected by numerous practical problems such as noisy sensor data, non-universality or lack of uniqueness of the chosen biometric feature, unacceptable error rates, and spoof attacks [18]. Multimodal biometric systems 
conquer most of these drawbacks by combining the proofs obtained from various sources [20] [19]. Multimodal biometrics has produced better accuracy [22] and population coverage, while reducing susceptibility to spoofing. The major feature to multimodal biometrics is the amalgamation of various biometric modality data at the feature extraction, matching score, or decision levels [21]. Numerous multimodal biometrics techniques and algorithms have been proposed by several researchers [23-30].

In our previous work [1], we have made use of LGXP feature as for face and iris-based multi-modal biometric recognition system. In this work, we have done a detailed analysis over our previous technique [1] with the technique given by Zhifang Wang et al. [2] who combined iris and face features for multi modal biometric recognition system using PCA and Gabor feature. The analysis is conducted over the two different set of face and iris image databases taken from CASIA and AT\&T. Here, the performances of the previous technique with LGXP-feature, LBP-based feature, EMD (Empirical Mode Decomposition) feature and the technique proposed by Zhifang Wang et al. [2] are extensively analyzed with the help of FMR and FNMR curve. Finally, ROC graphs in between the FNMR vs. FMR that are the two chief metrics used in this experimentation is drawn to demonstrate the effectiveness of the previous approach. The rest of the paper is organized as follows: Section 2 describes the description of the methods taken for analysis for iris and face recognition. Section 3 discusses about the results discussion and section 4 concludes the paper.

\section{DESCRIPTION OF THE METHODS TAKEN For ANALYSIS}

In the proposed approach, we compare the four feature extraction methods such as, Local Binary Pattern (LBP), local Gabor XOR pattern (LGXP), Empirical Mode Decomposition (EMD) and Principal Component Analysis (PCA) in face and iris-based multi modal recognition. The overall block diagram of our comparative analysis is shown in fig 1.

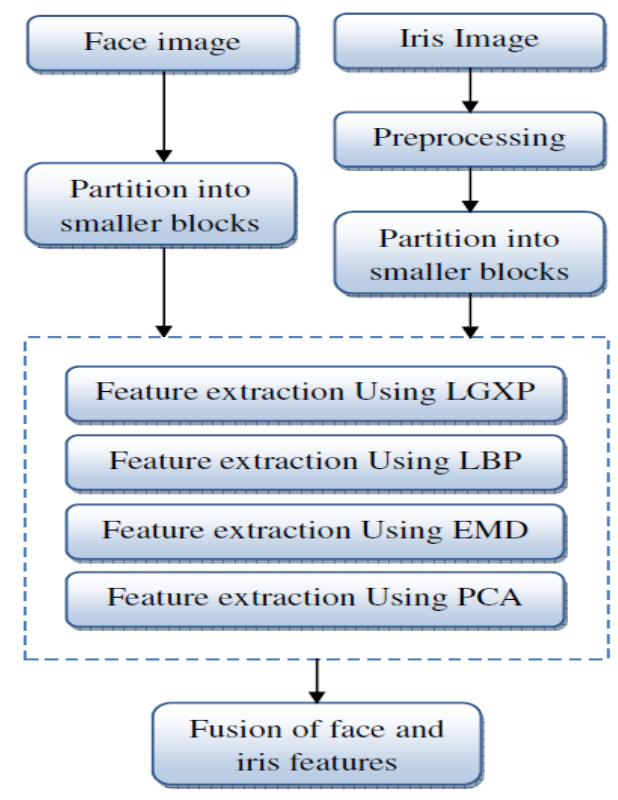

Fig.1: The Overall block diagram

\section{A. LGXP-feature-based face and iris recognition system}

In the first method, we make use of LGXP feature for multi-modal biometric recognition system. It consists of three modules such as, 1) Preprocessing 2) Feature Extraction of face and iris images and 3) Feature Matching.

\section{1) Preprocessing}

In the preprocessing stage, initially the iris image is converted into its normalized form via some preprocessing techniques before the feature extraction is made. The normalized form of the iris image is often utilized by the researchers to extract the features for iris recognition. Then, the iris segmentation is performed for identifying the iris boundary. Because, detecting the inner and outer boundaries of the iris texture is very crucial for effectual feature extraction. Integro-differential, Hough transform, and active contour model are some of the robust methods employed for detecting the boundaries. Eventually, a normalized iris image is obtained from the preprocessing step.

2) Feature Extraction of face and iris images:

In the second module, the normalized iris image and the facial image are partitioned into many small blocks and the pixel values in each block are converted into vector. Then, $1-U$ vector is applied to linear scaling and LGXP, which provides the feature vector. The irrelevant pixels (i.e., pixels in the eyelash and eyelid areas) in case of iris are calculated on each block for knowing the block importance. At last, we concatenate the feature vector of both the iris and face images and also the count of immaterial pixels of the iris image. The following steps depict the entire procedure of feature extraction of face and iris images:

- Partition the normalized iris image and the input face image into several small blocks.

- Convert the block of size $d_{1} \times d_{2}$ into $1-U$ vector.

- Perform LGXP on each block of both the normalized iris image and the input face image. In this stage, we apply LGXP to the normalized iris image as well as to the face image. LGXP is applied on each of the rescaled 1-U vector $D_{V}^{N}$ in order to acquire the feature of block $D$. After all the iterations, the LGXP creates a residual vector for both the iris and face images. The feature vector $F^{q}$ and $I^{q}$ (residual) of the block in face image and the iris image respectively, are represented as follows:

$$
\begin{aligned}
& F^{q}=\left(F_{1}^{q}, F_{2}^{q}, \ldots \ldots \ldots \ldots, F_{m}^{q}\right) \\
& I^{q}=\left(I_{1}^{q}, I_{2}^{q}, \ldots \ldots \ldots \ldots \ldots, I_{m}^{q}\right)
\end{aligned}
$$

Where, $F^{q}$ and $I^{q}$ represents the residual of the LGXP results of $D_{V}^{N}$.

- Combine the feature vector \& the count of immaterial pixels of the iris image and the feature vector of the face image.

- The resultant concatenated feature vector is given as, $\left.T^{q}=\left(F_{1}^{q}, F_{2}^{q}, \ldots \ldots \ldots \ldots, F_{m}^{q}, I_{1}^{q}, I_{2}^{q}, \ldots \ldots \ldots \ldots \ldots, I_{m}^{q}, C_{r}\right)\right)$ 
Where, $F^{q}$ and $I^{q}$ represents the residual of the LGXP results of $D_{V}^{N}$ for the face and iris image respectively and $C_{r}$ is the number of immaterial pixels for a block $D$.

\section{3) Feature Matching:}

The subsequent steps describe the process involved in the matching phase.

Calculation of Weightage Based on Irrelevant Pixels: The concatenated feature vector is computed for a test sample having both the iris and face images, and it is compared with the concatenated vectors of an iris image and face image of the database. For each block, we compute the block weightage based on the irrelevant pixels of test sample and an iris and face images of database.

Let the number of irrelevant pixels corresponding to the first block of a test sample be $C_{s}$ and the image of database be $C_{r}$. Then, the weightage $W_{y}$ of the first block is calculated using the formula given below,

$$
W_{y}= \begin{cases}1-\frac{C_{y}}{d_{1} \times d_{2}} ; & \text { if } \frac{C_{y}}{d_{1} \times d_{2}}<0.5 \\ 0 ; & \text { otherwise }\end{cases}
$$

Where, $C_{y}=\max \operatorname{imum}\left(C_{r}-C_{s}\right)$

Using the above equation, the blocks are obtained with three diverse set of values. $W_{y}=1$ represents the block without noise, $0.5<W_{y}<1$ represents the block with partial noise, and $W_{y}=0$ represents the block with noise. Likewise, we compute the block weightage for all the blocks with respect to test image and sample of the database.

Score Computation Using Distance Metrics: Here, Euclidean distance (ED) measure is adopted for the score computation. Selecting a suitable similarity measure for matching the feature vectors is vital and selection of distance measure compliments the proposed technique. This metric provides a score, which represents the similarity between two feature vectors (i.e., test sample and images from database). Therefore, for each block, the ED measure $S_{x}$ between the feature vectors say $T_{r}^{q}$ and $T_{t}^{q}$ is determined by using the following equation,

$$
S_{x}=E D\left(T_{r}^{q}, T_{t}^{q}\right)=\sqrt{\sum_{i=1}^{m}\left(T_{r_{i}}^{q}-T_{t_{i}}^{q}\right)^{2}}
$$

Average Matching Score Based on Weightage: In the prior steps, we have obtained a block weightage and matching score for all the blocks. These two values are then exploited to calculate the average matching score of the iris image. The formula used for computing the average matching score $M_{\text {avg }}$ is given below,

$$
M_{a v g}=\frac{\sum_{b=1}^{n} M_{b}}{\sum_{b=1}^{n}\left(1-W_{y_{b}}\right)}
$$

Where, $M=S_{x} \times\left(1-W_{y}\right)$

$M \rightarrow$ Weightage of block pair.

After performing the average matching of all the images, we obtain the value $\left(M_{\text {avg }}\right)$. Then, this value is employed to determine whether the test sample is already present in the database or not. If the obtained value $M_{\text {avg }}$ is below the predefined threshold $(P)$, then we can decide that the test sample is present in the database.

\section{B. LBP-feature-based face and iris recognition system}

In the second method, we make use of LBP feature for multi-modal biometric recognition system instead of LGXP feature. It consists of three modules such as, 1) Preprocessing 2) Feature Extraction of face and iris images and 3) Feature Matching.

i) Preprocessing: Initially, the input iris and face image is transformed to its normalized form. The above mentioned process is also used here to transform it to the normalized form.

ii) Feature Extraction of face and iris images: The whole procedure of feature extraction of face and iris images includes following steps: 1) Partitioning of Normalized Iris Image, 2) Conversion of Block of Size $d_{1} \times d_{2}$ into 1-U Vector, 3) Performing LBP on Each Block of both the Normalized Iris Image and the Input Face Image. 4) Concatenation of Feature Vector and the Count of Irrelevant Pixels of the Iris Image and the Feature Vector of the Face Image.

LBP operator: Local binary patterns were introduced by Ojala et al. [31] as a fine scale texture descriptor. In its simplest form, an LBP description of a pixel is created by thresholding the values of the $3 \times 3$ neighborhood of the pixel against the central pixel and interpreting the result as a binary number. Figure 2 illustrates the basic LBP operator.

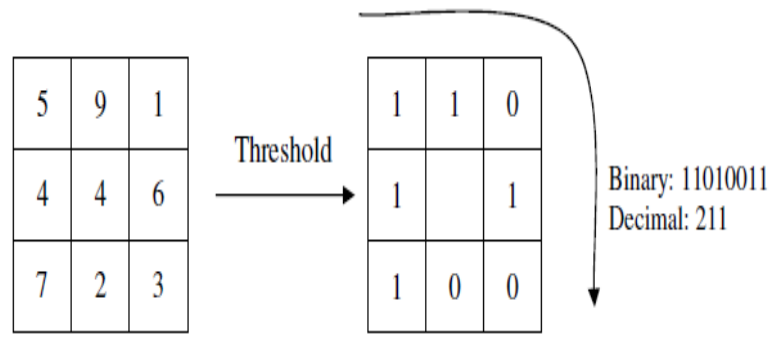

Fig.2: The basic LBP operator

3) Feature Matching: In feature matching, Weightage is computed for every block using the weightage equation defined above and matching Score is computed using the following formulae. 


$$
M_{a v g}=\frac{\sum_{b=1}^{n} M_{b}}{\sum_{b=1}^{n}\left(1-W_{y_{b}}\right)}
$$

Where, $M=S_{x} \times\left(1-W_{y}\right)$

$$
M \rightarrow \text { Weightage of block pair. }
$$

We obtain the value $\left(M_{\text {avg }}\right)$ after performing average matching of all the images. This value is used to decide whether the test sample is already present in the database or not. If the obtained value $M_{a v g}$ is less than the predefined threshold ( $P$ ), then the test sample is present in the database.

\section{EMD feature-based face and iris recognition system}

Here, we make use of Empirical Mode Decomposition (EMD) as feature for performing the multi-modal biometric recognition system. The remaining process (preprocessing and feature matching) is exactly same as that of previous step described in above sections.

Empirical Mode Decomposition: For the time-frequency analysis of real-world signals, an algorithm called empirical mode decomposition (EMD) is devised in [32]. By using EMD, the signal in hand is decomposed into numerous oscillatory modes called intrinsic mode functions (IMFs) [22, 26, 43]. Intrinsic Mode Functions (IMF): A function is an IMF, if and only if it satisfies two conditions: Condition 1: The number of extrema and the number of zero crossings differ by no more than one. Condition 2: At any point, the average of the envelope defined by the local maxima and by the local minima is zero. To decompose a signal by means of EMD, a sifting process [44] is used to extract the IMF from a given signal. Sifting Process: The procedure to decompose a signal $S(t)$, where $t=\{1,2, \cdots, T\}$ and $T$ is the total no. of samples in $S(t)$, is explained in detail as follows:

Step 1: Determine all the maxima points in the given signal $S(t)$ and generate the upper envelope say $S_{u p}(t)$ via Cubic Spline Interpolation.

Step 2: Determine all the minima points in the given signal $S(t)$ and generate the lower envelope say $S_{\text {low }}(t)$ via Cubic Spline Interpolation.

Step 3: Compute the mean of obtained upper envelope i.e., $S_{u p}(t)$ and lower envelope i.e, $S_{l o w}(t)$.

$$
N(t)=\frac{S_{u p}(t)-S_{\text {low }}(t)}{2}
$$

Step 4: The detail $D(t)$ of the given signal is obtained by taking the difference between the original signal $S(t)$ and the mean of the envelopes $N(t)$. $D(t)=S(t)-N(t)$
Step 5: Check whether the detail $D(t)$ is an IMF or not. Condition 1: If $D(t)$ have the same number of extrema and zero-crossings or can differ at most by one; Condition 2: If the average of $S_{u p}(t)$ and $S_{\text {low }}(t)$ is zero at any point.

Step 6: If the above two conditions are fulfilled, then $D(t)$ is an IMF. So, replace $S(t)$ by its residue $R(t)=S(t)-D(t)$. If both conditions are not fulfilled, then $D(t)$ is not an IMF and thus, replace $S(t)$ by $D(t)$.

Step 7: Repeat step 1 to 5 until it meets the termination criteria.

Stopping Criteria:- For determining a stopping criterion for the sifting process, a standard deviation (SD) is used. The $\mathrm{SD}$ is calculated from the two successive sifting results and is generally set between 0.2 and 0.3 .

$$
S . D=\sum_{t=1}^{T} \frac{\left|S_{n}(t)-S_{n+1}(t)\right|^{2}}{S_{n}^{2}(t)}
$$

\section{Zhifang Wang et al. [2] face and iris recognition system}

The fourth method taken for experimentation was proposed by Zhifang Wang et al. [2] who combined gabor and PCA features for recognition system. The detailed block diagram of the technique is given in figure 3 . In the first phase, the features of iris and face are extracted and then, normalization procedure was carried out for applying to the fusion process.

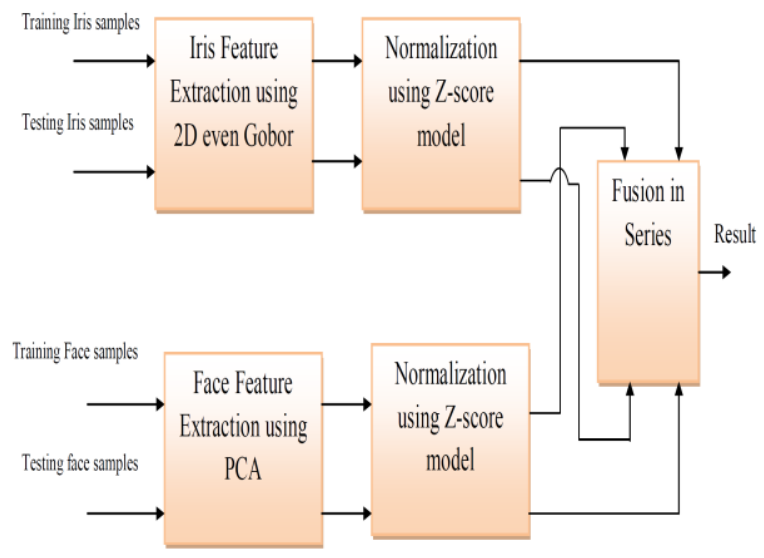

Fig. 3. Block diagram of the method proposed by Zhifang Wang et al. [2]

i) Face feature extraction: The following steps briefly portray the feature extraction method,

- Convert the matrixes of the face images into vectors as the training samples. 
- Calculate the mean of all training sample images, $\bar{x}$.

- Create total scatter matrix $S_{T}$,

$$
S_{T}=\sum_{i=1}^{n} \sum_{j=1}^{m}\left(x_{j}^{i}-\bar{x}\right)\left(x_{j}^{i}-\bar{x}\right)^{T}
$$

Where, $\bar{x}=\frac{1}{n m} \sum_{i=1}^{n} \sum_{j=1}^{m} x_{j}^{i}$ is the mean of all training sample images.

- Calculate the eigen values and the corresponding eigen vectors of $S_{T}$, and all eigen vectors compose the projection matrix $W_{P C A}$.

- Compute the projected feature vectors $y_{j}^{i}$,

$$
y_{j}^{i}=W_{P C A}^{T} x_{j}^{i}
$$

- Determine the Euclidean distance between the projected feature vectors and the template vectors, and perform the classification.

ii) Iris feature extraction: For iris recognition, a Gabor filter is used, which is one of the prominent feature extractors. We segment the portion of the iris after performing the preprocessing process. Then, the features from the iris are extracted via 2D even Gabor filter. Gabor function contains fine characteristic in direction and frequency. A self-similar multi-channel filter family is obtained through rotation, scaling, and translation of the Gabor function. The 2D even Gabor filter is defined below,

$$
g(x, y)=\frac{1}{2 \pi \sigma_{x} \sigma_{y}} \exp \left[-\frac{1}{2}\left(\frac{x^{2}}{\sigma_{x}^{2}}+\frac{y^{2}}{\sigma_{y}^{2}}\right)\right] \cdot \cos \left(2 \pi f \sqrt{x^{2}+y^{2}}\right)
$$

Where, $\sigma_{x}, \sigma_{y}$ are the parameters of scale, and $f$ represents the frequency. We partition the segmented iris image into sub-image with the same size, and correspondingly filter them by means of the Gabor filter family. The $j^{\text {th }}$ filtered sub-image is achieved by

$$
F_{j}(m, n) \iint I(x, y) g_{i}(m-x, n-y) d x d y
$$

Where, $g_{i}(x, y)$ is the $i^{\text {th }}$ even Gabor filter. Eventually, the absolute mean deviation is defined as the iris feature of the $j^{\text {th }}$ filtered sub-image.

$$
V_{j}=\frac{1}{N}\left(\sum_{N} F_{j}(m, n)-M\right)
$$

Here, $M$ is the mean value of the filtered sub-image, and $N$ is the number of the filtered sub-image. All the absolute mean deviation of the filtered sub-image composed the iris feature. However, the dimension of the iris feature derived by Tan's technique is very large. Hence, a PCA is employed to overcome this problem and control the dimension of the iris feature equal to that of the face feature.

iii) Normalization: Normally, the feature-level fusion techniques directly combine two kinds of features after feature extraction process. It is well-known that the order of magnitude and the distribution between iris feature and face feature might be different due to the dissimilarity of the modal and extraction technique. In order to eradicate the unbalance and to obtain better performance, before fusion, the features are normalized by means of z-score model. Let $a_{j}^{i}$ be a $d$ dimension iris feature of the $j^{\text {th }}$ iris training sample from the $i^{\text {th }}$ class, and $b_{j}^{i}$ be a $d$-dimension face feature of the $j$ th face training sample from the $i^{\text {th }}$ class. Subsequently, the iris feature set and the face feature set are represented as,

$$
A=\left(a_{1}^{1}, \ldots, a_{m}^{1}, a_{1}^{2}, \ldots, a_{m}^{n}\right) \text { and } B=\left(b_{1}^{1}, \ldots, b_{m}^{1}, b_{1}^{2}, \ldots, b_{m}^{n}\right)
$$

Let $A_{k}$ be the $k^{\text {th }}$ row of the iris feature set $A$. We use the following method to obtain the corresponding normalized component $X_{k}$. Initially, compute

$$
C_{k}=\frac{A_{k}-\bar{A}_{k}}{\sigma_{k}}
$$

Where, $\bar{A}_{k}$ represents the mean value of $A_{k}$, and $\sigma_{k}$ is the standard deviation of $A_{k}$. Then, we can obtain the normalized component by

$$
X_{k}=\frac{C_{k}-C_{\min }}{C_{\max }}
$$

Where, $C_{\min }$ and $C_{\min }$ are the minimum and maximum values of $C_{k}$ respectively. Finally, the normalized iris feature set is $X=\left(X_{1}, \ldots, X_{d}\right)$. Similarly for face feature, repeat the same process and acquire the normalized feature set $Y=\left(Y_{1}, \ldots, Y_{d}\right)$.

iv) Feature Fusion: As we know, $X=\left(X_{1}, \ldots, X_{d}\right)$ is a normalized iris feature vector, and $Y=\left(Y_{1}, \ldots, Y_{d}\right)$ is a normalized face feature vector. The fusion feature $\xi$ in sum rule can be defined as $\xi=\left(x_{1}+y_{1}, \ldots, x_{d}+y_{d}\right)$. For weighted sum rule, we take $\theta=\frac{3}{7}$ as the weighted parameter because the performance of iris recognition is superior compared to face recognition. The fusion feature in weighted sum rule is represented as $\xi=\left(x_{1}+\theta y_{1}, \ldots, x_{d}+\theta y_{d}\right)$. Thus, the sum rule can also be considered as a special case of weighted sum rule. In this paper, a series fusion technique is utilized. The format of the fusion feature is defined as $\xi=\left(x_{1} \ldots x_{d}, y_{1}, \ldots, y_{d}\right)$. This technique fuses two types of 
feature into a long vector. Subsequently, a Euclidean distance is selected to categorize the fusion features.

\section{RESULTS AND DISCUSSION}

The experimentation has been carried out on a $3.20 \mathrm{GHz}$ i5 PC machine with $8 \mathrm{~GB}$ main memory running on a 64-bit version of Windows 2007. In this section, we analyze the performance of different technique. In sub-section 4.1, describes dataset description for face and iris recognition. Subsection 4.2 describes the evaluation metrics using for our comparison work. The performance analysis results presented in sub-section 4.3 showed that the comparison results. The four techniques taken for comparative analysis are implemented in MATLAB.

\section{A. Dataset Description}

We have tested our approaches using two different datasets, namely CASIA and ORL. CASIA iris image database [3] includes 756 iris images from 108 eyes (hence 108 classes). For each eye, 7 images are captured in two sessions, where three samples are collected in the first session and four in the second session. In ORL database, there are ten different images of each of 40 distinct subjects. Face images and the Iris images from the databases are shown in figure 4 and 5 respectively.
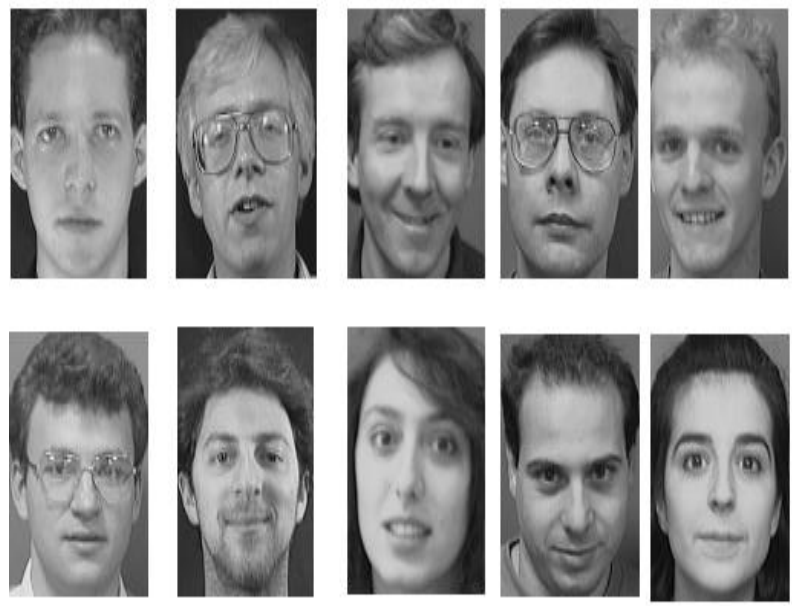

Fig. 4. Face images taken from ORL face database
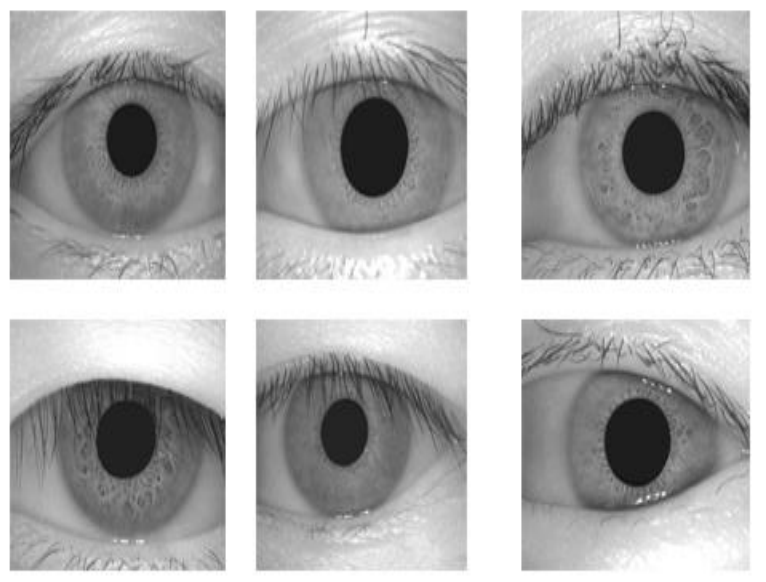

Fig. 5. Iris images taken from CASIA iris database

\section{B. Evaluation Metrics}

False Match Rate (FMR):- The False Match Rate (FMR) shows the proportion of persons who were falsely accepted during the characteristics comparison. Those efforts that were previously refused (Failure to Acquire, FTA) due to a low quality (e.g. of the image), contrary to FAR (False acceptance rate), are not taken into consideration. It depends on the application whether a falsely accepted feature contributes to increase the FAR or FRR (False rejection rate).

False Non-Match Rate (FNMR):- The False Non-Match Rate (FNMR) shows the proportion of persons who were falsely not accepted during the characteristics comparison. Those efforts that were previously rejected (Failure to Acquire, FTA) due to a low quality (e.g. of the image), contrary to FRR, are not taken into consideration. Again, it relies on the application whether a falsely non-accepted feature contributes to increase the FRR or FAR.

ROC curve: The FMR(t) (False Match Rate) and $\operatorname{FNMR}(t)$ (False Non-Match Rate) are calculated for $t$ ranging from 0 to 1 . Then, the ROC curve is plotted FMR vs. FNMR for varying threshold $t$. The plotted ROC curve is extensively used in the contest to compare the performance of different algorithms. One more parameter used for comparison is, Equal Error Rate ( $E E R$ ) that is computed as the point where $F N M R(t)=F M R(t)$.

\section{Performance Analysis}

1) Analysis of the LGXP-based Approach for Features Fusion in Face and Iris recognition

The performance analysis of the LGXP is presented in this sub-section. For various threshold values, FMR and FNMR are computed. Then, the graph is plotted for the computed values to find the efficiency in acceptance of the genuine user and the rejection of the impostor user for different threshold levels. The performance analysis graph on LGXP method is shown in figure 6. From the plotted graphs in figure 6, we have found the equal error rate (EER) of the LGXP- approach is given as, $\mathrm{EER}=0.5$.

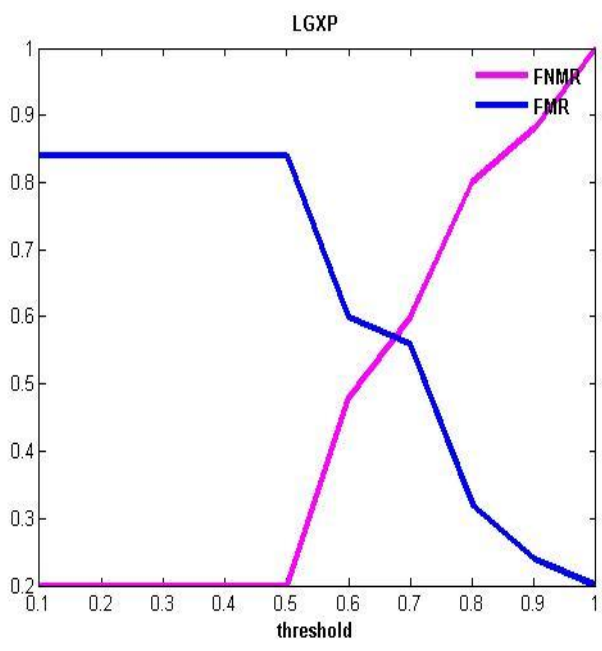

Fig. 6. Performance analysis graph for LGXP 
2) Analysis of the LBP-based Approach for Features Fusion in Face and Iris recognition

The performance analysis of the LGXP-based approach is presented in this sub-section. For various threshold values, FMR and FNMR are computed. Then, the graph is plotted for the computed values, shown in figure 7 . From the plotted graphs in figure 7, we have found the equal error rate (EER) of the LBP-based approach is given as, $\mathrm{EER}=0.5$.

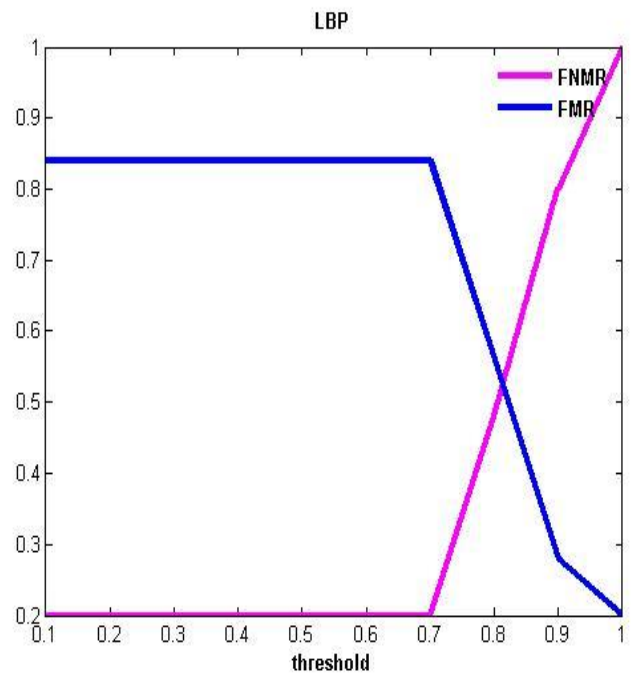

Fig. 7. Performance analysis graph for LBP

3) Analysis of the EMD-based Approach for Features Fusion in Face and Iris recognition

This section presents the performance plot of the EMDbased approach in face and iris recognition. For various thresholds, the FMR and FNMR is computed for the same approach and the computed values are used to plot graph. The graph shown in figure 8 is the performance of the EMD-based approach such a way, the EER of the technique is computed from the plotted graph, EER=0.56.

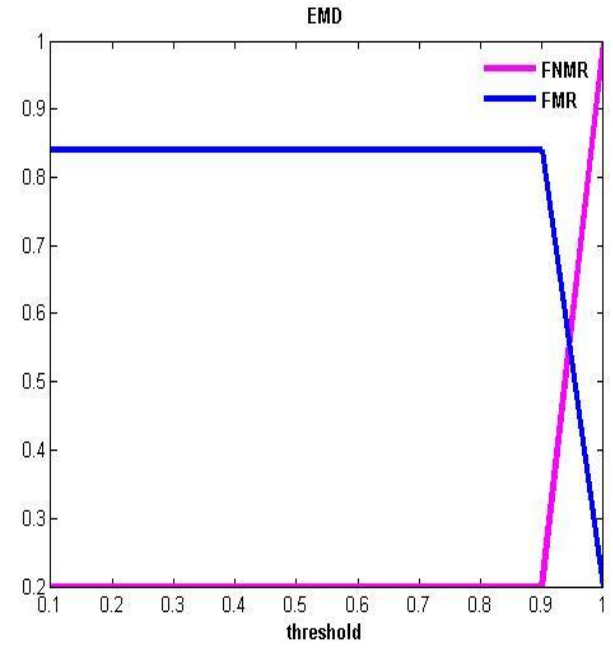

Fig. 8 Performance analysis graph for EMD
4) Analysis of the PCA-based Approach for Features Fusion in Face and Iris recognition

The performance analysis graph of PCA-based technique is shown in figure 9 . From the plotted graphs shown in figure 9, we have found the equal error rate (EER) of the approach, $\mathrm{EER}=0.57$ which is high compared with other three techniques.

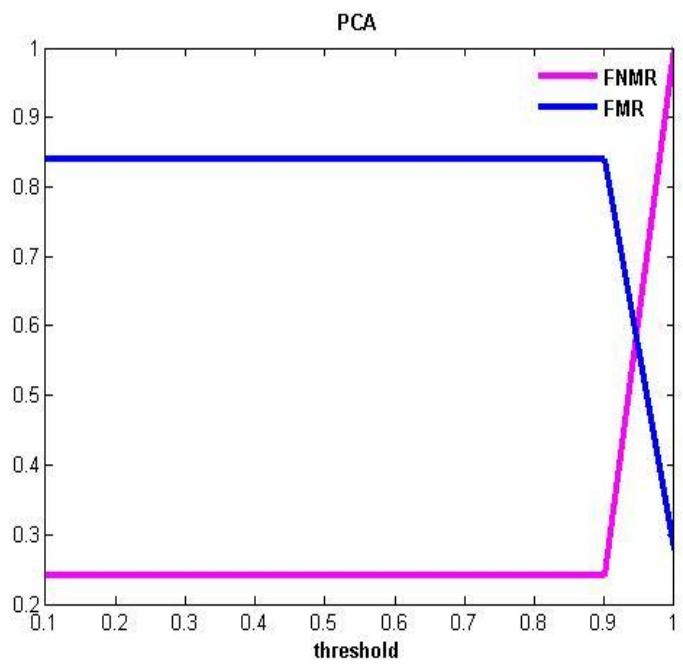

Fig. 9. Performance analysis graph for PCA

\section{Comparative Analysis}

The comparative analysis of four multimodal approaches such as LGXP, LBP, EMD and PCA is performed using the ROC curve. The plotted ROC curve for the comparative analysis is given in the figure 10. From the ROC curve graph, we have analyzed that the LGXP and LBP-based method has a lower FNMR value which means the better security of the proposed multimodal biometric system. When compared with the PCA method, the proposed three approaches provided better results.

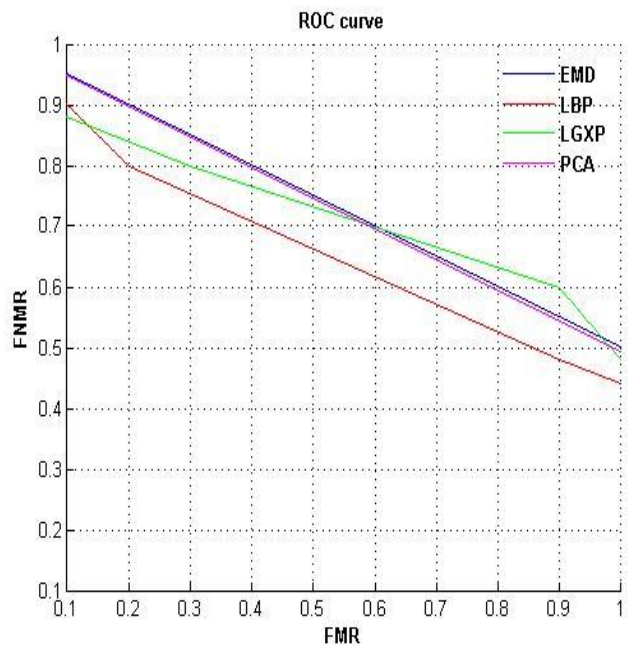

Fig. 10 ROC curve graph for four methods 


\section{CONCLUSION}

We have presented comparative analysis of four different feature extraction approaches, such as LBP, LGXP, EMD and PCA. The major processes of the four methods are consists of 1) Feature extraction from face image, 2) Feature extraction from iris image and 3) Fusion of face and iris features. The performance of the feature extraction methods in multi-modal recognition was analyzed using FMR and FNMR to study the recognition behavior of these approaches.

Then, an extensive analysis was carried out to find the effectiveness of different approaches using ROC curve. For experimentation, we have used the CASIA iris image database and the ORL face database and the evaluation results clearly demonstrated that the performance of the LBP and LGXP method is better compared with PCA-based technique.

\section{REFERENCES}

[1] Deepak Sharma and Dr. Ashok Kumar "Multimodal Biometric Recognition System: Fusion of Face and Iris Features Using Local Gabor Patterns", International Journal of Advanced Research in Computer Science (IJARCS), Volume 2, Number 6, Nov-Dec 2011.

[2] Zhifang Wang, Erfu Wang, Shuangshuang Wang and Qun Ding, "Multimodal Biometric System Using Face-Iris Fusion Feature", journal of computers, vol. 6, no. 5, May 2011.

[3] Chinese Academy of Sciences - Institute of Automation (CASIA), Database of 756 Gray scale Eye Images, http://www.cbsr.ia.ac.cn/IrisDatabase.htm,Version 1.0, 2005.

[4] Hayasaka, A.Shibahara, T.Ito, K.Aoki, T. Nakajima and H. Kobayashi, "A 3D Face Recognition System Using Passive Stereo Vision and Its Performance Evaluation", In proceedings of International Symposium on Intelligent Signal Processing and Communications, pp.379 - 382, 2006.

[5] Ching-Han Chen and Chia TeChu, "Fusion of Face and Iris Feature for Multimodal Biometrics", Lecture Notes in Computer Science, Vol. 3832, pp. 571-580, 2005.

[6] Sanjay Kumar Mohanty and Prasant Kumar Pattnaik, "Authentication Based on Texture Analysis and SVM Classification", International Journal of Instrumentation, Control \& Automation (IJICA), Vol.1, No.1, pp. 61-66, 2011.

[7] A. Ross and A. K. Jain, "Information fusion in biometrics, "Pattern Recognition Letters, Vol. 24, pp. 2115-2125, Sep 2003.

[8] L. I. Kuncheva, C. J. Whitaker, C. A. Shipp, and R. P. W. Duin, "Is independence good for combining classifiers?," in Proc. of Int'l Conf. on Pattern Recognition(ICPR), vol. 2, pp. 168-171, 2000.

[9] Arun Ross and Anil K. Jain, "Multimodal Biometrics: An Overview", in Proc. of 12th European Signal Processing Conference (EUSIPCO), pp.1221-1224, September 2004.

[10] Pascal Paysan, ReinhardKnothe, Brian Amberg, Sami Romdhani and Thomas Vetter, "Face Recognition Using 3-D Models: Pose and Illumination “, In proceedings of IEEE, Vol. 94, No.11, pp.1977 - 1999, 2009.

[11] H B Kekre and V ABharadi, "Gabor Filter Based Feature Vector for Dynamic Signature Recognition", International Journal of Computer Applications, Vol. 2, No.3, pp.74-80, May 2010.

[12] A. Jain, L Hong and S Pankati. "Biometric Identification". Communications of The ACM, vol. 43, no. 2, pp. 90-98. Feb. 2000.

[13] J.G.Daugman, "High Confidence Visual Recognition of Persons by a Test of Statistical Independence", IEEE Transactions on Pattern Analysis and Machine Intelligence, Vol.15, No.11, pp. 1148-1161, 1993 .

[14] Sandipan P. Narote, Abhilasha S. Narote and Laxman M. Waghmare,
"Iris Based Recognition System using Wavelet Transform", International Journal of Computer Science and Network Security, Vol.9, No.11, November 2009.

[15] Sudha Gupta, Viral Doshi, Abhinav Jain and SreeramIyer "Iris Recognition System using Biometric Template Matching Technology", International Journal of Computer Applications, Vol.1, No.2, pp.21-30, 2010.

[16] Srinivasa Kumar devireddy, "An Accurate Human Identification through Iris recognition", Georgian electronic scientific Journal in Computer Science and Telecommunication, Vol.6, No. 23, pp. 22-29, 2009.

[17] Prof G.Ramaswamy VudaSreenivasarao, Dr.P.Ramesh, D.RaviKiran, "A Novel Approach for Human Identification through Fingerprints", International Journal of Computer Applications, Vol.4, No.3, pp. 35-42, July 2010.

[18] Z.Yaghoubi, K.Faez, M.Eliasi and A.Eliasi, "Multimodal biometric recognition inspired by visual cortex and Support vector machine classifier", International Conference onMultimedia Computing and Information Technology (MCIT), pp.93 - 96, 2010.

[19] Anil Jain, Karthik Nandakumar and Arun Ross, "Score normalization in multimodal biometric systems", Pattern Recognition, Vol. 38, No.12, pp.2270-2285, Dec. 2005.

[20] L. Hong, A.K. Jain, S. Pankanti, “ Can multibiometrics improve performance? ", In proceedings of IEEE Workshop on Automatic Identification Advanced Technologies,pp.59-64,1999.

[21] Robert Snelick, Umut Uludag, Alan Mink, Michael Indovina, and Anil Jain, Large-Scale Evaluation of Multimodal Biometric Authentication Using State-of-the-Art Systems", IEEE Transactions on Pattern Analysis and Machine Intelligence, Vol. 27, No. 3, pp.250-255, March 2005.

[22] A.K. Jain, R. Bolle, and. Pankanti, "Biometrics: Personal Identification in Networked Society", 1999.

[23] ShufuXie, ShiguangShan, XilinChenand Jie Chen, "Fusing Local Patterns of Gabor Magnitude and Phase for Face Recognition", IEEE Transactions on Image Processing, Vol. 19, No. 5, pp.1349-1362, May 2010.

[24] JieLin, Jian-Ping Li, Hui Lin and Ji Ming,“ Robust person identification with face and iris by modified PUM method", International Conference on Apperceiving Computing and Intelligence Analysis, pp. 321-324, 2009.

[25] Jun-Ying GanandJun-Feng Liu, "Fusion and recognition of face and iris feature based on wavelet feature and KFDA", International Conference on Wavelet Analysis and Pattern Recognition, pp.47-51, 2009.

[26] Shoa'aJadAllah Al-Hijaili and ManalAbdul Aziz, "Biometrics In Health Care Security System, Iris-Face Fusion System", International Journal of Academic Research, Vol. 3, No. 1., pp.11-19, January, 2011.

[27] Baochang Zhang, Shiguang Shan, XilinChenand Wen Gao, "Histogram of Gabor Phase Patterns (HGPP): A Novel Object Representation Approach for Face Recognition", IEEE Transactions on Image Processing, Vol. 16, No. 1, pp.57-68, January 2007.

[28] HarinSellahewa and Sabah A. Jassim, "Image-Quality-Based Adaptive Face Recognition", IEEE Transactions on Instrumentation and Measurement, Vol. 59, no. 4, pp.805-813, April 2010.

[29] Zhenan Sunand Tieniu Tan, "Ordinal Measures for Iris Recognition", IEEE Transactions on Pattern Analysis and Machine Intelligence, Vol.31, No.12, pp. 2211 - 2226, 2009

[30] Ryan N. Rakvic, Bradley J. Ulis, Randy P. Broussard, Robert W. Ives and Neil Steiner, "Parallelizing Iris Recognition", IEEE Transactions on Information Forensics and Security, Vol. 4, No. 4, pp. 812- 823, December 2009.

[31] Ojala, T., Pietik"ainen, M., Harwood, "D.: A comparative study of texture measures with classification based on feature distributions," Pattern Recognition, vol. 29, pp.51-59, 1996.

[32] D. J. Duffy, "The Application Of Hilbert-Huang Transforms To Meteorological Datasets", Journal of Atmospheric and Oceanic Technology, vol. 21, no. 4, pp.599-611, 2004. 